\title{
EFECTO DE LA CÁSCARA SOBRE EL MECANISMO DE ENDURECIMIENTO DEL FRIJOL COMÚN (Phaseolus vulgaris): RESULTADOS PRELIMINARES1
}

\author{
L. F. de León, R. Bressani y L.G. Elías ${ }^{2}$
}

\section{COMPENDIO}

Frijol negro (Phaseolus vulgaris), variedad Tamazulapa fue utilizado para conocer los cambios físicos y químicos que ocurren durante el almacenamiento del frijol con o sin cáscara y asî́ determinar el papel que juega la cáscara en este proceso. Se prepararon muestras de cotiledones y de frijol entero, las cuales se almacenaron durante 6 semanas a $37^{\circ} \mathrm{C}$ y $90 \%$ de humedad relativa, al final de las cuales la muestra del frijol entero se dividió en dos sub-muestras. Una de ellas quedó Intacta, y la otra se decorticó manualmente. La cinética de endurecimiento para las tres muestras fue totalmente diferente y el grano con cáscara se endureció a una mayor velocidad. El tiempo de cocción (TC) del frijol entero aumentó de 99 minutos a más de 480 minutos en seis semanas. El de los cotiledones aumentó de 45 a 111 minutos. En el período de las seis semanas el TC de los cotiledones almacenados como semilla entera, pero evaluados sin la cáscara aumentó de 45 a 103 minutos. En el grano entero y en los cotiledones la absorción de agua disminuyó, aunque la disminución fue mucho mayor en los cotiledones, probablemente por la gran capacidad de absorción que tiene la cáscara. Como resultado del almacenamiento, los componentes químicos fueron modificados encontrándose una disminución en el contenido de pectatos solubles y aumento de los Insolubles. Disminución significativa en el contenido de taninos (ácido tánico) y cambios en los componentes de fibra dietética. Los datos obtenidos sugieren que la cáscara juega un significativo rol en el proceso de endurecimiento del frijol durante el almacenamiento.

Palabras clave adicionales: Cotiledón, almacenamiento.

\section{INTRODUCCIÓN}

El presente trabajo fue realizado con el objeto de determinar el papel que juega la cáscara sobre el mecanismo de endurecimiento del frijol y establecer los cambios físicos y químicos ocurridos tanto en el frijol con cáscara, como en el cotiledón y en la cáscara propiamente dicha, durante el almacenamiento. Con esto se pretende contribuir a aclarar el problema de endurecimiento del frijol, para posteriormente buscar mecanismos para tratar de resolver dicho problema.

\section{MATERIALES Y MÉTODOS}

\section{Materiales}

Para la realización del presente trabajo se utilizó frijol común (Phaseolus vulgaris) variedad Tamazulapa

\begin{abstract}
Samples of cotyledons and whole black beans (Phaseolus vulgaris, Tamazulapa variety) were stored for 6 weeks at $37^{\circ} \mathrm{C}$ and $90 \%$ relative humidity conditions in order to establish the chemical and physical changes and the role of the seed coat in the hard-to-cook phenomena. After the storage period, samples of whole beans were divided In two sub-samples: one with the and other without the seed coat. These two samples and the cotyledons were analyzed for cooking time, water absorption, dietary fiber, tanic acid, soluble pectins and phytic acid. Cooking time of whole beans Increased from 99 to 480 minutes; for the cotyledons, this value Increased from 45 to 111 minutes. Cooking time of the dehulled bean, stored as a whole bean, increased from 45 to 103 minutes. Water absorption in whole beans and cotyledon decreased, although In cotyledons this was higher. No changes were observed in the dietary fiber content of the cotyledons and the beans dehulled after storage. However, in the whole grains neutral -detergent fiber decreased, while acid detergent fiber, cellulose and lignin did not have a significant change. On the other hand, soluble pectates decreased In the whole bean and cotyledon; tannin content as tanic acid decreased only In the whole beans. The data obtained suggest that the seed coat could play a significant role in the hard-to-cook effect before and during storage.
\end{abstract}

cosecha 1987 , manteniendo en cuarto frío a una temperatura de $4^{\circ} \mathrm{C}$ hasta el momento de su uso.

\section{Métodos}

\section{Eliminación de la cáscara del frijol}

La cáscara del frijol fue eliminada manualmente con el objeto de no desgastar el grano de frijol, al igual que para no separar los dos cotiledones del mismo.

\section{Almacenamiento de las muestras}

Tanto frijol con cáscara como frijol descascarado (sólo cotiledón de frijol) fueron empacados en bolsas de polietileno y almacenados en desecadoras con una humedad relativa de $90 \%$. Las desecadoras fueron

\footnotetext{
'1 Presentado en la XXXV Reunión Anual del PCCMCA, San Pedro Sula, Honduras, 1989.

'2 Científicos, División Ciencias Agrícolas y de Alimentos, INCAP, Apartado Postal 1188, Guatemala, Guatemala, C.A. Publicación INCAP E-1291. Publicado en Agronomía Mesoamericana Vol. 1 (1990).
} 
puestas en cuarto de temperatura controlada, la cual estuvo a $41^{\circ} \mathrm{C}$ para mantener la temperatura dentro de la desecadora constante en un valor de $37^{\circ} \mathrm{C}$. Las muestras permanecieron en estas condiciones por un tiempo de 6 semanas, después del cual fueron sacadas, analizadas y conservadas en cuarto frío a $4^{\circ} \mathrm{C}$.

\section{Análisis físicos y químicos}

a. Análisis físicos: Se determinó el tiempo de cocción utilizando el Cocinador Mattson, la dureza del frijol mediante el Ottawa Texture Measuring y la absorción de agua medida después de poner en remojo el fríjol en agua durante 4 horas.

b. Análisis químicos: Se determinaron los componentes de la fibra dietética siguiendo el método propuesto por Goering y Van Soest (1970). Los polifenoles fueron determinados como ácido tánico y los pectatos solubles e insolubles: Mediante el método recomendado por Dietz y Rouse (citado por Kon (1968). Se determinó $\mathrm{Na}, \mathrm{Ca}, \mathrm{Mg}$ y $\mathrm{K}$ mediante absorción atómica, las soluciones de cenizas fueron preparadas siguiendo el método de la AOAC (1975).

\section{RESULTADOS Y DISCUSIÓN}

El Cuadro 1 muestra el efecto del almacenamiento del fríjol bajo condiciones drásticas de temperatura y humedad relativa sobre su tiempo de cocción. Lo primero que se aprecia de este cuadro es el efecto que tiene la cáscara sobre el tiempo de cocción del frijol. Esto significa que para el caso de este tipo de frijol fresco, la cáscara contribuye con cerca del $55 \%$ del tiempo de cocción del frijol, lo que indica que la cáscara es la primera barrera para la cocción del frijol y es responsable en gran medida por la dureza o el largo tiempo de cocción del frijol.

Por otro lado, en este Cuadro 1, se puede ver que el frijol con cáscara tiene un comportamiento muy diferente al comportamiento del cotiledón, es decir, que el frijol con cáscara tiene una velocidad de endurecimien-

Cuadro 1. Tiempo de cocción (minutos) de frijol fresco y endurecido cocinado en el Mattson

\begin{tabular}{lccc}
\hline & \multicolumn{3}{c}{ Tiempo de Almacenamiento (semana) } \\
\cline { 2 - 4 } Tipo de Frijol & $\mathbf{0}$ & $\mathbf{4}$ & $\mathbf{6}$ \\
\hline $\begin{array}{l}\text { Con cáscara } \\
\begin{array}{l}\text { Sin cáscara } \\
\text { (sólo cotiledón) }\end{array}\end{array}$ & $99 \pm 1 \mathrm{c}$ & $247 \pm 61$ & $>480 \mathrm{a}$ \\
$\begin{array}{l}\text { Sin cáscara, } \\
\text { (sólo cotiledón) }\end{array}$ & $45 \pm 3 \mathrm{~d}$ & $86 \pm 1$ & $111 \pm 1 \mathrm{~b}$ \\
\hline
\end{tabular}

1/ Frijol almacenado con cáscara y descascarado para análisis. Letras diferentes denotan diferencias estadísticamente significativa $(\mathrm{P}<0.05)$ to mayor, comparada a la velocidad de endurecimiento del cotiledón, igualmente el cotiledón del frijol almacenado con cáscara y descascarado para análisis, se endureció menos que el cotiledón solo, esto puede ser apreciado más fácilmente en la Figura 1, en la cual se muestra la cinética de endurecimiento del frijol con y sin cáscara. Lo anterior muestra el papel que juega la cáscara en el mecanismo de endurecimiento del frijol.

Al analizar los datos del tiempo de cocción del frijol, se observa que en el frijol con cáscara el tiempo de cocción aumentó más de 380 minutos, mientras que para el caso del cotiledón, el aumento en el tiempo de cocción fue únicamente de 65 minutos, esto indica que la cáscara posiblemente tuvo un endurecimiento equivalente a más de 320 minutos de cocción, En base a estos resultados, se infiere que en el frijol pueden estar ocurriendo dos tipos de endurecimiento: Uno a nivel de la cáscara, el cual posiblemente, sea el más significativo, y el otro a nivel del cotiledón.

Al final del período de almacenamiento, se encontró que la cáscara contribuyó con un $78.5 \%$ del tiempo de cocción del frijol, lo que muestra un aumento significativo si comparamos con la contribución que la cáscara tuvo en el tiempo de cocción del frijol fresco, el cual fue de $55 \%$.

Por último, en el Cuadro 1 se muestra el tiempo de cocción del cotiledón de frijol almacenado con cáscara, es decir, este frijol fue almacenado con cáscara y descascarado para análisis. Es importante indicar que este cotiledón tuvo un tiempo de cocción ligeramente menor al reportado por cotiledón almacenado sin cáscara. Esto viene

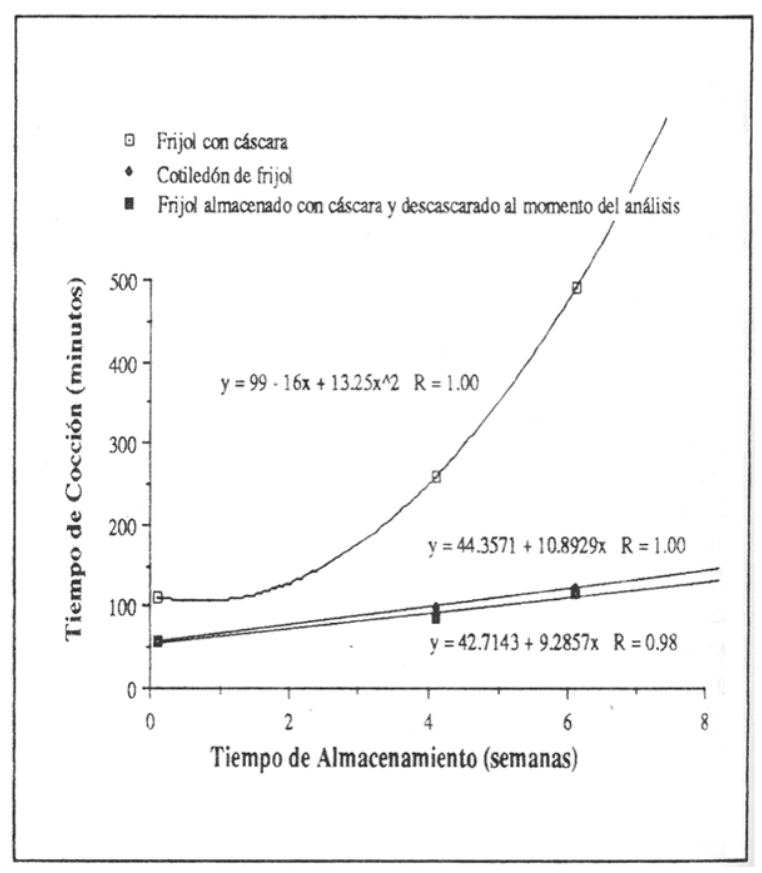

Figura 1. Cinética de endurecimiento del frijol común y sin cáscara almacenados a $37^{\circ} \mathrm{C}$ y $90 \% \mathrm{HR}$. 
a confirmar lo dicho anteriormente en cuanto a que el mayor grado de endurecimiento del frijol está ocurriendo a nivel de la cáscara, posiblemente la cáscara tiende a volverse más resistente (elástica) o más impermeable al agua, conforme el frijol permanece almacenado.

El Cuadro 2 sumariza los resultados de absorción de agua del frijol. Es importante mencionar que la capacidad de absorción de agua del frijol disminuyó como resultado del almacenamiento del grano y cuando se eliminó la cáscara del frijol antes del almacenamiento, la capacidad de absorción de agua disminuyó drástica y significativamente. Es interesante observar también que el cotiledón del frijol que fue almacenado con cáscara y descascarado para análisis presentó una mayor absorción de agua comparado al cotiledón endurecido, lo cual muestra claramente una buena relación entre absorción de agua y dureza o tiempo de cocción del frijol, lo que permite inferir que la pérdida de la capacidad de absorción de agua está muy ligada al problema de endurecimiento del frijol. Finalmente, en este Cuadro se muestra la pérdida de la capacidad de absorción de agua por parte de la cáscara durante el almacenamiento, es decir, que la cáscara de frijol endurecido absorbe menos agua, comparado a la que absorbe la cáscara de frijol fresco. Esto indica que en la cáscara pueden estar sucediendo algunas reacciones relacionadas con el mecanismo de endurecimiento del frijol.

Para tratar de determinar que compuestos del frijol están involucrados en el mecanismo de endurecimiento, se determinaron los componentes de la fibra dietética, los resultados son reportados en el Cuadro 3. Es de interés indicar que para el caso del cotiledón la fibra neutro detergente disminuyó y fue la más afectada por el almacenamiento. Los cambios que ocurrieron en el citoledón dentro de la cáscara del frijol fueron diferentes a los que ocurrieron en el frijol con cáscara, ya que por ejemplo, la fibra neutro detergente disminuyó grandemente cuando se analizó sólo cotiledón, mientras que para frijol con cáscara esta fracción de la fibra tendió a aumentar y las otras fracciones como son la fibra ácido

Cuadro 2. Absorción del agua del frijol común sometido a almacenamiento acelerado

\begin{tabular}{lcc}
\hline \multirow{2}{*}{ Tipo de Frijol } & \multicolumn{2}{c}{ Tiempo de Almacenamiento (semanas) } \\
\cline { 2 - 3 } & $\mathbf{0}$ & $\mathbf{6}$ \\
\hline $\begin{array}{l}\text { Con cáscara } \\
\text { Sin cáscara }\end{array}$ & $96.3 \pm 0,6 \mathrm{~b}$ & $91.3 \pm 1.8 \mathrm{c}$ \\
(sólo cotiledón) & $95.4 \pm 0.8 \mathrm{~b}$ & $59.3 \pm 0.9 \mathrm{e}$ \\
$\begin{array}{l}\text { Sin cáscara" } \\
\text { (sólo cotiledón) }\end{array}$ & $95.4 \pm 0.8 \mathrm{~b}$ & $72.9 \pm 2.0 \mathrm{~d}$ \\
Cáscara de frijol & $374.1 \pm 37.5 \mathrm{a}$ & $323.4 \pm 34.1 \mathrm{a}$ \\
\hline
\end{tabular}

1/ Frijol almacenado con cáscara y descascarado para análisis. Letras: diferentes denotan diferencias estadísticamente significativas $(\mathrm{P}<0.05)$.
Cuadro 3. Cambios en la composición de la fibra dietética como resultado del almacenamiento del frijol

\begin{tabular}{lcccc}
\hline & \multicolumn{5}{c}{ Componente de la Fibra Dietética (mg/ 100 g) } \\
\cline { 2 - 5 } Tipo de Frijol & $\begin{array}{c}\text { Fibra neutro Fibra Acido } \\
\text { detergente }\end{array}$ & $\begin{array}{c}\text { detergente } \\
\text { Lignina }\end{array}$ & Celulosa \\
\hline Cotiledón fresco & $18.73 \mathrm{e}$ & 2.90 & 0.99 & 2.55 \\
Cotiledón endurecido & $17.78 \mathrm{e}$ & 2.93 & 0.84 & 3.44 \\
$\begin{array}{l}\text { Cotiledón endurecido1 } \\
\text { Frijol con cáscara fresco }\end{array}$ & $27.50 \mathrm{~d}$ & 3.62 & 1.08 & 2.65 \\
$\begin{array}{l}\text { Frijol con cáscara } \\
\text { endurecido }\end{array}$ & 9.08 & 2.81 & 6.42 \\
$\begin{array}{l}\text { Cáscara de frijol fresco } \\
\text { Cáscara de frijol }\end{array}$ & $52.27 \mathrm{a}$ & 46.27 & 14.32 & 32.93 \\
endurecido & $50.49 \mathrm{~b}$ & 46.49 & 14.98 & 32.41 \\
\hline
\end{tabular}

1/ Frijol almacenado con cáscara y descascarado para análisis Letras diferentes denotan diferencias estadísticamente significativas $(\mathrm{P}<0.05)$

detergente, la lignina y la celulosa disminuyeron, contrario a lo ocurrido sólo para cotiledón.

Analizando los cambios ocurridos, únicamente en la cáscara se puede observar que en la cáscara de frijol endurecido, la fibra neutro detergente fue la que más cambios mostró tendiendo a disminuir, mientras que la celulosa aumentó ligeramente.

Estos resultados parecen indicar que la fibra dietética y sus componentes pueden estar jugando un importante papel en el endurecimiento del frijol, ya que posiblemente algunos de estos componentes como por ejemplo la fibra neutro detergente, pueden estar reaccionando con otros compuestos del frijol.

Con base a los resultados discutidos previamente, se puede concluir preliminarmente que la cáscara juega un importante papel en el mecanismo de endurecimiento del frijol y que existen dos tipos de endurecimiento en el frijol: Uno a nivel de la cáscara y otro a nivel del cotiledón, siendo el endurecimiento de la cáscara el de mayor significancia.

Es importante indicar que el contenido de taninos, expresados como ácido tánico (Cuadro 4), disminuyó como resultado del almacenamiento del frijol. Puede verse en este Cuadro que los taninos presentes en la cáscara disminuyeron durante el almacenamiento y que el cotiledón tendió a ganar estos taninos, esto muestra el papel de la cáscara y el de los taninos en el endurecimiento del frijol.

En el Cuadro 5 se muestran los cambios ocurridos en los pectatos contenidos en el frijol. Los resultados obtenidos indican que los pectatos solubles en agua disminuyen con el almacenamiento del frijol y por el contrario, los pectatos insolubles (solubles en EDTA) tienden a aumentar, esto confirma hipótesis previas sobre uno de los mecanismos del endurecimiento del frijol $(3,6)$. Es importante indicar que en el frijol con cáscara los cambios en pectatos solubles fueron mínimos, sin embargo, el cambio en los pectatos insolubles fue muy grande. 
Cuadro 4. Taninos (como ácido tánico en $\mathrm{mg} / \mathrm{g}$ materia seca) en frijol común antes y después del almacenamiento acelerado

\begin{tabular}{lcc}
\hline \multirow{2}{*}{ Tipo de Frijol } & \multicolumn{2}{c}{ Tiempo de Almacenamiento (semanas) } \\
\cline { 2 - 3 } & $\mathbf{0}$ & $\mathbf{6}$ \\
\hline $\begin{array}{l}\text { Con cáscara } \\
\text { Sin cáscara } \\
\text { (sólo cotiledón) }\end{array}$ & $3.28 \pm 0.23 \mathrm{e}$ & $1.64 \pm 0.06 \mathrm{e}$ \\
$\begin{array}{l}\text { Sin cáscara1/ } \\
\text { (sólo cotiledón) }\end{array}$ & $1.03 \pm 0.23 \mathrm{f}$ & $2.22 \pm 0.13 \mathrm{~d}$ \\
Cáscara de frijol & $19.48 \pm 0.23 \mathrm{f}$ & $1.40 \pm 0.00 \mathrm{e}$ \\
\end{tabular}

1/ Frijol almacenado con cáscara y descascarado para análisis. Letras diferentes denotan diferencias estadísticamente significativas $(\mathrm{P}<0.05)$.

Finalmente, en el Cuadro 6 se muestra el efecto del almacenamiento y el papel que puede tener la cáscara sobre el contenido de minerales del frijol. Es importante indicar que el contenido de sodio ( $\mathrm{Na}$ ) disminuyó significativamente en la cáscara durante el almacenamiento, esto parece confirmar estudios previos realizados por de León (2) en el sentido de que este mineral puede estar jugando un rol importante en el fenómeno de endurecimiento del frijol.

En base a los resultados obtenidos se puede concluir que la cáscara juega un papel importante en el proceso de endurecimiento del frijol y que es en esta parte anatómica donde pueden estar ocurriendo los mayores cambios, por lo tanto, es importante tomar en cuenta esta parte del grano al realizar los diferentes ensayos físicos y químicos, principalmente los relacionados a la determinación del tiempo de cocción y dureza de i grano.

\section{BIBLIOGRAFIA}

ASSOCIATION OF OFFICIAL AGRICULTURAL CHEMISTS. 1975. Washington, D.C. Official Methods of Analysis of the Association of Agricultural Chemists. 12th. ed. Washington, D.C.

DE LEON, L.F. 1987. Soluciones salinas: Una tecnología económica para, la utilización del frijol común (P. vulgaris) endurecido. Tesis (Maestro), USAC. Facultad de Ciencias Químicas y Farmacia, INCAP/CESNA. Curso

Cuadro 5. Contenido de pectatos (g/100 g muestra) en frijol común antes y después del almacenamiento acelerado.

\begin{tabular}{|c|c|c|c|c|}
\hline \multirow{2}{*}{ Tipo de Frijol } & & \multicolumn{3}{|c|}{ Pectatos solubles en } \\
\hline & & Agua & EDTA & Hidróxido de Sodio \\
\hline \multirow[t]{2}{*}{ Con cáscara } & Fresco & $1.16 \pm 0.16 \mathrm{~b}$ & $0.10 \pm 0.02 \quad \mathrm{f}$ & $1.58 \pm 0.01$ \\
\hline & Endurecido & $0.85 \pm 0.10 \quad b$ & $1.29 \pm 0.01 \mathrm{e}$ & $2.15 \pm 0.13 \mathrm{hi}$ \\
\hline \multirow[t]{2}{*}{ Sin cáscara (sólo cotiledón) } & Fresco & $2.14 \pm 0.13 \mathrm{a}$ & $0.13 \pm 0.05$ & $1.19 \pm 0.28$ \\
\hline & Endurecido & $1.00 \pm 0.07 b$ & $0.21 \pm 0.03$ & $0.88 \pm 0.19$ \\
\hline \multirow[t]{2}{*}{ Sin cáscara ${ }^{1 /}$ (sólo cotiledón) } & Fresco & $2.14 \pm 0.13 \mathrm{a}$ & $0.13 \pm 0.05$ & $1.19 \pm 0.28$ \\
\hline & Endurecido & $1.14 \pm 0.02$ & $0.05 \pm 0.01 \quad \mathrm{f}$ & $0.96 \pm 0.31$ \\
\hline \multirow[t]{2}{*}{ Cáscara de frijol } & Fresca & $0.90 \pm 0.00 \mathrm{~b}$ & $0.36 \pm 0.02 \mathrm{e}$ & $3.66 \pm 0.20$ \\
\hline & Endurecida & $1.56 \pm 0.00 \mathrm{~b}$ & $0.42 \pm 0.02 \mathrm{~d}$ & $3.07 \pm 0.13 \mathrm{gh}$ \\
\hline
\end{tabular}

1/ Frijol almacenado con cáscara y descascarado para análisis.

Letras diferentes denotan diferencias estadísticamente significativas $(\mathrm{P}<0.05)$.

Cuadro 6. Contenido de sodio y potasio (mg/100 g muestra) en frijol común (P. vulgaris) antes y después del almacenamiento acelerado.

\begin{tabular}{lccccc}
\hline & \multicolumn{4}{c}{ Tipo de Almacenamiento (semanas) } \\
\cline { 2 - 3 } \cline { 5 - 6 } Tipo de Frijol & $\mathbf{0}$ & $\mathbf{K}$ & & $\mathbf{N a}$ & $\mathbf{6}$ \\
\hline Con cáscara & $61.23 \pm 2.1 \mathrm{~b}$ & $1335 \pm 2.12 \mathrm{~d}$ & & $60.68 \pm 5.4 \mathrm{~b}$ & $1358 \pm 24.8 \mathrm{~cd}$ \\
Sin cáscara (sólo cotiledón) & $64.43 \pm 7.7 \mathrm{~h}$ & $1434 \pm 18.40 \mathrm{e}$ & & $59.25 \pm 6.0 \mathrm{~b}$ & $1481 \pm 4.2 \mathrm{c}$ \\
Sin cáscara 1/ (sólo cotiledón) & $64.43 \pm 7.7 \mathrm{~b}$ & $1434 \pm 18.40 \mathrm{e}$ & & $58.77 \pm 5.5 \mathrm{~h}$ & $1488 \pm 26.2 \mathrm{e}$ \\
Cáscara de frijol & $93.16 \pm 0.1 \mathrm{a}$ & $662 \pm 0.70 \mathrm{e}$ & & $63.87 \pm 8.6 \mathrm{~b}$ & $723 \pm 25.5 \mathrm{e}$ \\
\hline
\end{tabular}

1/ Frijol almacenado con cáscara y descascarado para análisis.

Letras diferentes denotan diferencias estadísticamente significativas $(\mathrm{P}<0.05)$. 
de postgrado en Ciencia y Tecnología de Alimentos. Guatemala. p. 93.

GOERING, H.K.; VAN SOEST, P.J. 1970. Forage fiber analysis. Agriculture Handbook No. 379. Agricultura Research Service. United States Department of Agriculture. USA.

HOLM, et al. 1986. Starch. 36(7):224-226.
KON,S. 1968. Pectic substances of dry beans and their possible correlation with cooking time. J. Food Sci. $33: 436-438$.

MOSCOSO, W. 1981. Efecto del almacenamiento a alta temperatura y alta humedad sobre algunas características físicas y químicas del frijol. Presentado en: XXVII Reunión Anual del PCCMCA. Santo Domingo, República Dominicana, 23-27 marzo 1981. 